Case Report

\title{
Pheochromocytoma: a mimicry of ACS
}

\begin{abstract}
There are diseases which at times presents in such a way as to mimic for an acute coronary syndrome event. Pheochromocytoma is one of those disorders. Episodic or transient hypertension is one of its cardinal features. Presence of palpitation, diaphoresis and headache along with hypertension alarms for Pheochromocytoma. However, accompanying of these symptoms with a family history of Pheochromocytoma or syndromic presentation of MEN 2A, MEN 2B, VHL or Neurofirbomatosis, further increases the likelihood. Therefore, should be screened with a more sensitive test like plasma levels for catecholamine and metanephrine. Scanning techniques should be carried out to localize the tumor and to look for malignancy. Once localized, the only curative therapy is surgical resection of the tumor.
\end{abstract}

Volume 3 Issue 3 - 2015

Haseeb Ashraf

King Edward Medical University, Pakistan

Correspondence: Haseeb Ashraf, King Edward Medical University, Pakistan Registrar Cardiac ICU at AI-Dar Hospital, Madina Munawwara, Neela Gumbad, Anarkali, Lahore, Pakistan, Tel 923219200037,Email styledmussel@hotmail.com

Received: February 17, 2015 | Published: September 04, 2015

\section{History}

A 36yrs old female admitted in coronary care unit through emergency department with history of chest pain, heaviness in interscapular region, radiating to back of head and left arm. She also experienced episodic palpitations and vomiting. Previously she is known to be hypertensive with history of episodic rise of blood pressure which has been occurring for the last 8 years. Her past history is also positive of similar episodes with palpitations, vomiting and dizziness. Each episode lasting for up to 5-10minutes and relieving spontaneously. Episodes were usually precipitated by salt and sweet intake.

\section{Examination}

On examination her blood pressure was $160 / 110 \mathrm{mmHg}$, with regular pulse of $100 / \mathrm{bpm}$. Normal heart sounds with no evidence of gallop rhythm or murmur. Her mental system examination was grossly intact. Abdominal examination showed soft and no tenderness. Her systemic examination was unremarkable. ${ }^{1}$

\section{Diagnosis / Differential diagnosis}

a. Acute coronary syndrome

b. Malignant hypertension

c. Syncope

d. Pheochromocytoma

\section{Investigations and interventions}

a. ECG showed sinus tachycardia with asymmetrical, deep T-wave inversions in leads 1, II, avL and V2-6.

b. Echocardiography showed normal sized LV and global hypokinesia with echogenic myocardium more of anteroapical segments. LV EF was $48 \%$.

c. Her lab studies showed normal blood count, fasting lipid profile, liver function tests, thyroid function test. Her cardiac enzymes and troponin $\mathrm{T}$ turned out to be normal.

d. 2days after admission patient's chest pain settled but she complained of headache and nausea. Repeat ECG showed asymmetrical, deep T-wave inversions with prolong QT-interval (Figure 1). ${ }^{2}$

Her ultrasound abdomen was performed as a routine investigation which revealed a right adrenal mass. This finding was further confirmed on CT abdomen. Later, her urinary vinyl mendalic acid levels came out to be raised.

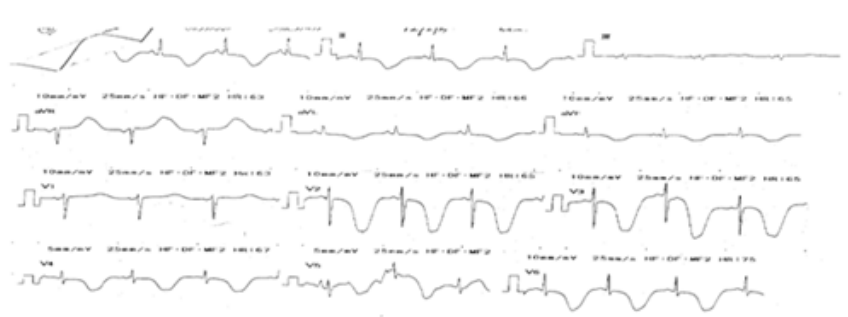

Figure I Coronary angiography was performed with surprisingly normal coronaries.

\section{Treatment}

Her surgery was planned for the removal of right adrenal mass followed by histopathological examination of the mass. Histopathology report showed pheochromocytoma of malignant nature.

\section{Discussion}

Pheochromocytoma is a rare tumor named after its dusky colored appearance after histological staining. It is a catecholamine secreting disorder. Norepinephrine is the most commonly secreted agent followed by epinephrine but however, in rare instances dopamine and vasoactive intestinal peptide is also observed. Its characteristics are known by the rule of 10 .

a. $10 \%$ are malignant (rest are benign).

b. $10 \%$ involve both the adrenal glands.

c. $10 \%$ occur in children

d. $10 \%$ occur extra adrenal (anywhere from brain to urinary bladder).

e. $10 \%$ occur in children.

f. $10 \%$ are diagnosed after an even of stroke.

g. $10 \%$ are familial.

h. $10 \%$ are not associated with hypertension. ${ }^{3}$

\section{History and physical}

Most common symptoms accompanying phechromocytoma are palpitaitons, headache and diaphoresis along with hypertension. However, other symptoms which may be present are;

a. Tremulousness

b. Dizziness 


\section{c. Syncope \\ d. Weight loss \\ e. Chest pain \\ f. Nervousness}

Rarely patients may also present with diarrhea if the secreting agent in the tumor is vasoactive intestinal peptide (VIP).

On physical examination the most important finding of all would be the elevated blood pressure. Hypertension is transient in 50\% of the cases and may be persistent finding. Hypertension may be severe enough to cause encephalopathy. Sinus tachycardia is the commonest form of finding among arrhythmias associated with pheochromocytoma but however, ventricular tachycardia has also been observed in some patients which may or may not be accompanied with dilated cardiomyopathy, Other physical findings could be
i. Neurofibromas
ii. Café au let spots
iii. Diabetes
iv. Goiter
v. Tachycardia and tachyarrhythmias
vi. Retinopathy
vii. Fever
viii. Cardiomyopathy
ix. Pulmonary edema

\section{Etiology and associated syndromes}

Pheochromocytoma which occur in the hereditary form are associated most commony with following syndromes
a. MEN 2A.
b. MEN 2B.
c. Von Recklinghausan disease or Neurofibromatosis.
d. Von Hipple Lindau (VHL) syndrome (Table 1). ${ }^{4,5}$

Table I Characteristic diagnostic features of the above listed syndrome

\begin{tabular}{|c|c|c|c|}
\hline MEN 2A & MEN 2B & Neurofibromatosis & VHL syndrome \\
\hline \multicolumn{3}{|c|}{ Medullary Thyroid CAMedullary thyroid CA Neurofibromas } & Pheochromocytoma \\
\hline \multirow{4}{*}{$\begin{array}{l}\text { Parathyroid Adenoma } \\
\text { Pheochromocytoma }\end{array}$} & Pheochromocytoma & Pheochromocytoma & Cerebellar \\
\hline & Hirschsprung disease & & hemangioblastoma \\
\hline & Mucosal & & Renal cell CA \\
\hline & neurofibromatosis & & Renal \& pancreatic \\
\hline \multirow{4}{*}{$\begin{array}{l}\text { Hisrschsprung } \\
\text { Disease }\end{array}$} & Intestinal & & cyst \\
\hline & ganglio & & Frididvmal \\
\hline & neurofibromatosis & & Epidioymal \\
\hline & Marfanoid body & & cystadenoma \\
\hline
\end{tabular}

\section{Diagnosis}

Diagnostic test of choice for detection of catecholamine and degradation products (metanephrine) depends upon the probable likelihood of pheochromocytoma. In high risk patients who presents either with the family history or genetic syndrome should be tested with plasma catecholamine and metanephrine levels. Plasma testing for catecholamine has high specificity of $96 \%$ with a relatively decreased specificity. However, those patients who are not high risk for the suspicion of pheochromocytoma should be tested with 24hour urinary catecholamine and metanephrine levels. Its diadnostic specificity is highest with $99.7 \%$. CT or MRI scan should be used for detection of adrenal masses. MRI has $100 \%$ sensitivity in detecting adrenal pheochromocytoma tumors. Scintigraphy is carried out in cases where CT and MRI scanning is unable to localize the tumor. PET scan is another technique which can efficiently localize the tumor in pheochromocytoma. Once the diagnosis has been confirmed, genetic testing for various familial syndromes may be done in cases where the suspicion is there. ${ }^{6}$

\section{Management}

Curable treatment for pheochromocytoma is surgical resection. Laproscopic tumor removal is indicated in localized, small tumors with easy approach. Open laprotomy is indicated in conditions where laproscopic approach is not feasible or the tumor is larger in size. In most of the cases symptoms wears off after surgery however, special attention should be paid before hand in avoiding hypotension due to abrupt removal of catecholamines from the blood after surgery. Therefore, patient is advised adequate hydration and salt intake. Medically patient with pheochromocytoma and hypertension should be managed towards control of blood pressure in order to avoid dire consequences. Beta blockers are only indicated after alpha blockade has been effectively introduced for at least 2 days. Only then beta blockers should be initiated which otherwise would have lead to unopposed vasoconstriction and hypertensive crisis. Before diagnostic laboratory testing is performed certain medications should be avoided which may interfere with the results. Medications which interfere with the results are betablockers, levodopa, tricyclic antidepressants, chlorpromazine, amphetamine and methyldopa. Certain medications may also precipitate hypertensive crisis like TCAs and metochlopromide. Pheochromocytoma in pregnancy is quite rare but once present has grave prognosis. It has significantly high morbidity and mortality affecting both the mother and fetus.

\section{Prognosis}

Patient left untreated or with malignancy has poor prognosis. Malignant pheochromocytomas have 5year expected survival rate of almost $50 \%$. In benign conditions, 5years survival is up to $95 \%$. Cardiovascular manifestations which are commonly encountered and produce worse prognosis are;

\section{a. Atrial and ventricular fibrillation}

b. Myocarditis

c. Symptoms and signs mimicking myocardial infarction.

d. Tako tsubo cardiomyopathy

e. Pulmonary edema

\section{f. Hypertensive crisis}

Pregnancy is another condition which with pheochromocytoma poses life threatening consequences. Hypertensive crisis in pheochromocytoma may lead to devastating neurologic consequences like intracerebral ischemic or hemorrhagic stroke and hypertensive encephalopathy. Surgical removal of the catecholamine producing tumor almost cures the disorder.

\section{Learning points}

Every chest pain with features suggestive of anginal pain does not necessarily means the presence of coronary artery disease, no 
matter how grave the electrocardiographic changes may appear Pheochromocytoma is one of the diseases which mimics for myocardial infarction or broadly speaking acute coronary syndrome. Tako tsubo cardiomyopathy is increasingly getting more fame and could be a presenting feature. A detailed history should raise the suspicion for pheochromocytoma even though it is a rare disorder Ruling out for pheochromocytoma is important because its resection almost cures the disease completely.

One should be well aware of the syndromic associations of pheochromocytoma.

\section{Acknowledgments}

None.

\section{Conflicts of interest}

Author declares there are no conflicts of interest.

\section{Funding}

None.

\section{References}

1. Tucker ME. Endocrine Society first to address rare adrenal-tumor care. Medscape Medical News. 2014.

2. Lenders JW, Duh QY, Eisenhofer G, et al. Pheochromocytoma and paraganglioma: an endocrine society clinical practice guideline. J Clin Endocrinol Metab. 2014;99(6):1915-1942.

3. Sheps SG, Jiang NS, Klee GG, et al. Recent developments in the diagnosis and treatment of pheochromocytoma. Mayo Clin Proc. 1990;65(1):88-95.

4. Därr R, Lenders JWM, Hofbauer LC, ET AL.Pheochromocytoma: Update on Disease Management. Ther Adv in Endo and Metab. 2012;3(1):11-26.

5. PrejbiszA, Lenders JW, Eisenhofer G, etal. Cardiovascularmanifestations of phaeochromocytoma. J Hypertens. 2011;29(11):2049-2060.

6. Eisenhofer G, Lenders JW, Timmers H, et al. Measurements of plasma methoxytyramine, normetanephrine, and metanephrine as discriminators of different hereditary forms of pheochromocytoma. Clin Chem. 2011;57(3):411-420. 